\title{
Performance Analysis of Various Schemes for True Images Compression using GUI in MATLAB
}

\author{
Ashish Kumar \\ Singh \\ M.Tech Scholar ECE \\ Department SHIATS \\ DU Allahabad, India
}

\author{
Mukesh Kumar \\ Assistant Professor \\ ECE Department \\ SHIATS-DU Allahabad, \\ India
}

\author{
A.K. Jaiswal \\ Professor, H.O.D. ECE \\ Department SHIATS- \\ DU Allahabad, India
}

\author{
Rohini Saxena \\ Assistant Professor \\ ECE Department \\ SHIATS-DU Allahabad, \\ India
}

\begin{abstract}
Image compression is a widely addressed researched area. Image compression is a key technology in transmission and storage of digital images because of vast data associated with them. Various types of compression methods are present in place. The performance analysis of various schemes to compress a True image in graphical user interface is discussed in this paper. True images can be compressed with the help of same scheme as grayscale images by applying them to each of the three colour components. Experimental results demonstrate that the STW wavelet algorithm is more efficient for PSNR while EZW method provides the better compression ratio.
\end{abstract}

\section{Keywords}

Image compression, DWT, EZW, SPIHT, STW, PSNR, MSE, CR.

\section{INTRODUCTION}

Image compression is important for many applications that involve large data storage, transmission and retrieval. Image compression is nothing but reducing the amount of data required to represent an image. Original images need huge storage capacity. Image compression technique is use to reduce redundancy in image. The reduction of file size allows more images to be stored in a limited memory space. To analysis the parameters of image compression PSNR and CR is important parameters. It gives synthetic performance of the compression of images. Image is a 2 Dimensional signal represented by Digital system. Normally Image taken from the camera is in the analog form. However for processing, transmitting and storage, images are converted in to digital form. A digital Image is basically 2- Dimensional array of pixels. Basically compressing an image is different from compressing digital data. Traditional image compression techniques have been designed to exploit the statistical redundancy present within real world images. The discrete cosine transforms (DCT), DPCM, and the entropy coding of Subband images are examples of this statistical approach. Removing redundancy can only give a limited amount of compression, to achieve high ratios; some of non-redundant information must be removed.

\section{MEAN SQUARE ERROR}

MSE is a risk function, corresponding to the expected value of the squared error loss or quadratic loss. The average of the square of the difference between the desired response and the actual system output.
$\mathrm{MSE}=\frac{1}{M N} \sum_{Y=1}^{M} \sum_{X=1}^{N}\left[I(X, Y)-I^{\prime}\left(X^{\prime}, Y^{\prime}\right)\right]^{2}$

Where $\mathrm{m} \mathrm{x} \mathrm{n}$ is the size of the image and $\mathrm{I}(\mathrm{X}, \mathrm{Y})$ is the original input image and I' $\left(X^{\prime}, Y^{\prime}\right)$ is the compressed image.

PEAK SIGNAL TO NOISE RATIO PSNR is an approximation to human perception of reconstruct ion quality. PSNR defined the ratio of maximum possible power and the power of corrupting noise in an image. A higher PSNR indicates higher quality of reconstruction. The PSNR is defined as-

$\mathrm{PSNR}=20 * \log 10(255 /$ sqrt $(\mathrm{MSE})$

\section{TRUE COLOR IMAGES}

True color is the specification of the color of a pixel on a display screen using a 24-bit value, which allows the possibility of up to $16,777,216$ possible colors. A pixel's color is defined by number of bits usually called as bit depth. True color images have 24-bit to represent three RGB colors which use to represent and store image information like colors, hues, shades.

\section{IMAGE COMPRESSION PROCESS}

Fig. 1 describes the fundamental steps of an image Compression system. The image compression system consists two basic structural blocks: an encoder and a decoder ${ }^{[5]}$. Encoder creates some symbols from the input data to represent the image. Image $\mathrm{f}^{n}(\mathrm{x}, \mathrm{y})$ represent an approximation of the input data that results from compressing and subsequently decompressing the input image.

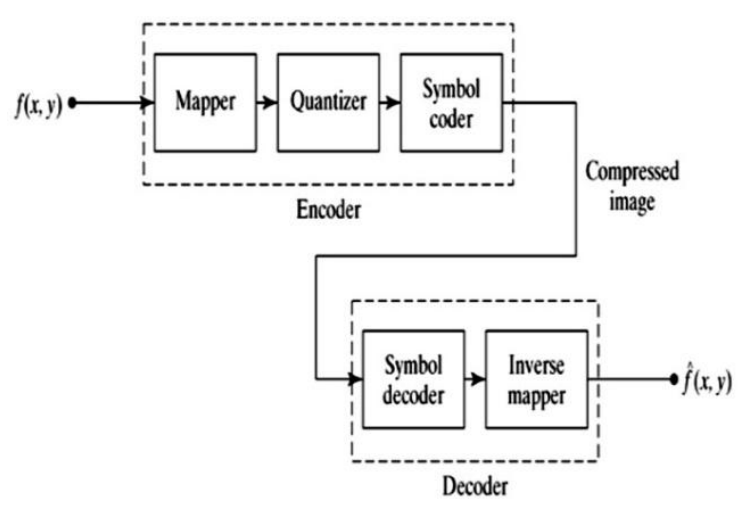

Fig.1 Basic steps in an image compression system. 
The formula of compression ratio is following:

$\mathrm{CR}=\mathrm{n} 1 / \mathrm{n} 2$

Where $\mathrm{n} 1$ represent the bits of information carrying in the original image and $\mathrm{n} 2$ denote the bits of information carrying in the compressed image respectively. A compression ratio of 10:1 denotes that the original image has 10 information bits for every 1 bit in the compressed image.

\section{PRINCIPLE AND PROCESS OF WAVELET TRANSFORM}

Wavelets are local in time and scale and generally have an irregular shape. A wavelet waveform has limited duration and an average value of zero. Some wavelets show a property ideal for compact signal representation called orthogonality. This property can fix the problem of over representation. A wavelet transform can decompose a signal into component wavelets and scaled representations of the original mother wavelet ${ }^{[1]}$. Once this is done the coefficients of the wavelets can be decimated to remove some of the details. Small Wavelets are able to separate the fine details in a signal, while large wavelets can recognize the coarse details.

A wavelet function $\Psi(\mathrm{t})$ has two main properties,

$$
\int_{-\infty}^{0} \Psi(t) d t=0
$$

That is, the function is oscillatory or has wavy appearance.

$$
\int_{-\infty}^{0}|\Psi(t)|^{2} d t<\infty
$$

That is, the most of the energy in $\Psi(\mathrm{t})$ is confined to a finite duration.

\section{2D DISCRETE WAVELET TRANSFORM}

The 2D wavelet transform uses the 'mother wavelet transform' technique but needs an extra step at each level of decomposition ${ }^{[2]}$. The 2-D DWT is carried out by applying the 1-D DWT in both the horizontal and the vertical direction of the image. 2D images are generally matrices with $\mathrm{N}$ rows and $\mathrm{M}$ columns. Any type of decomposition of an image into wavelets involves a pair of waveforms-

1. One to represent the high frequency corresponding to the detailed part of the image (wavelet function).

2. One for low frequency or smooth parts of an image (scaling function).

The horizontal data of image is filtered during each level of decomposition, and then the approximation and information produced from this are filtered on columns. In each step the approximation, the vertical, the horizontal and the diagonal types of details of images are obtained ${ }^{[3]}$. Multiplication of scaling function $(\varphi(\mathrm{x}, \mathrm{y}))$ and wavelet functions $(\Psi(\mathrm{x}, \mathrm{y}))$ provide the wavelet function in 2D discrete wavelet transform. We get following four details of image after first level of decomposition,
Approximate details $-\Psi(\mathrm{x}, \mathrm{y})=\varphi(\mathrm{x}) \varphi(\mathrm{y})$

Horizontal details $-\Psi(\mathrm{x}, \mathrm{y})=\varphi(\mathrm{x}) \Psi(\mathrm{y})$

Vertical details $-\Psi(\mathrm{x}, \mathrm{y})=\Psi(\mathrm{x}) \varphi(\mathrm{y})$

Diagonal details $-\Psi(\mathrm{x}, \mathrm{y})=\Psi(\mathrm{x}) \Psi(\mathrm{y})$

The approximation details can then be put through a filter bank, and this is repeated until the required level of decomposition has been reached ${ }^{[4]}$. The filtering step is followed by a sub-sampling operation that decreases the resolution from one transformation level to the other.

After applying the 2-D filler bank at a given level $\mathrm{n}$, the detail coefficients are output, while the whole filter bank is applied again upon the approximation image until the desired maximum resolution is achieved. Fig. 2 shows wavelet filter decomposition. The sub-bands are labelled by using the following notations,

1. LLn represents the approximation image nth level of decomposition, resulting from low pass filtering in the vertical and horizontal both directions.

2. LHn represents the horizontal details at nth level of decomposition and obtained from horizontal lowpass filtering and vertical high-pass filtering.

3. HLn represents the extracted vertical Details/edges, at nth level of decomposition and obtained from vertical low-pass filtering and horizontal high-pass filtering.

4. HHn represents the diagonal details at nth Level of decomposition and obtained from High pass filtering in both directions.

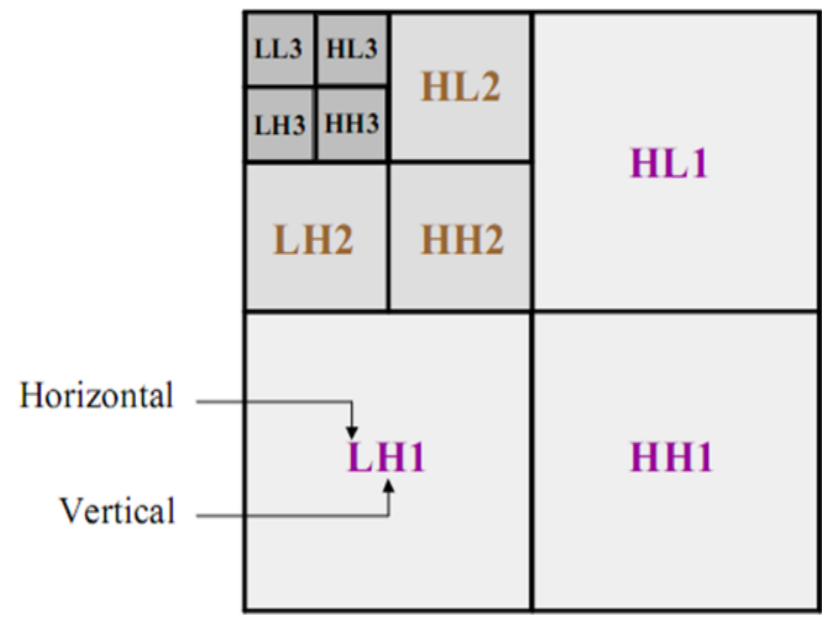

Fig.2 Wavelet Filter Decomposition 


\section{METHODOLOGY}

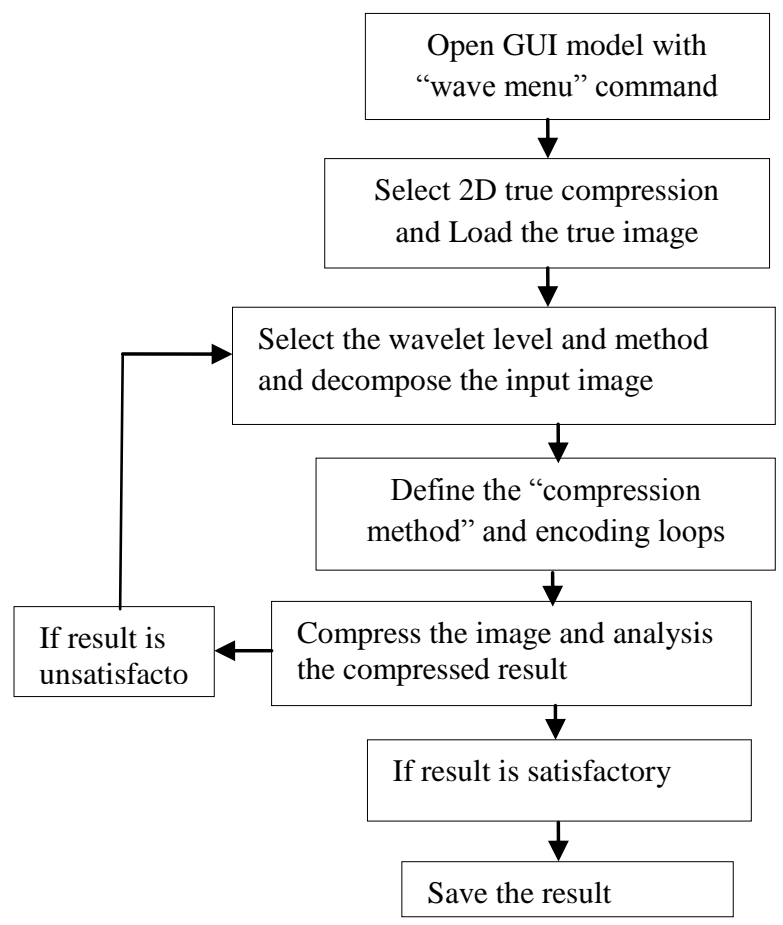

Flow chart of true compression using GUI model

\section{RESULT \& DISCUSSION}

True image compression using GUI can be done through Simulink library. Here firstly choose the true color images and then perform the image compression process in wavelet toolbox. The following image compression results perform the comparison between SPIHT, EZW and STW wavelet methods. Each method has its own specification. Table no.1 shows the difference between each method. Following experimental result show that STW method gives the better PSNR (55.02) and MSE (0.2046) value while EZW method provides the better compression ratio $(11.94 \%)$.

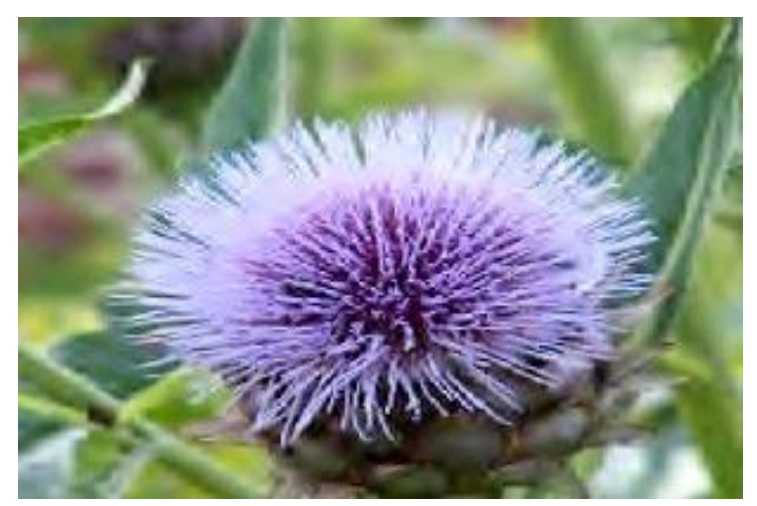

Fig.3. Original image

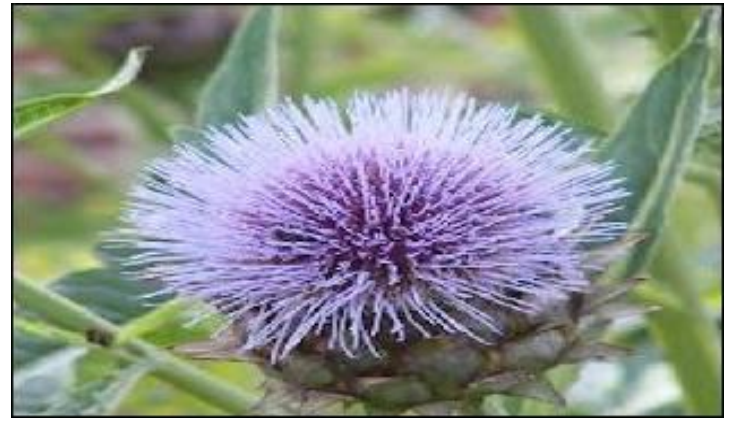

Fig.4. Compressed image by EZW method

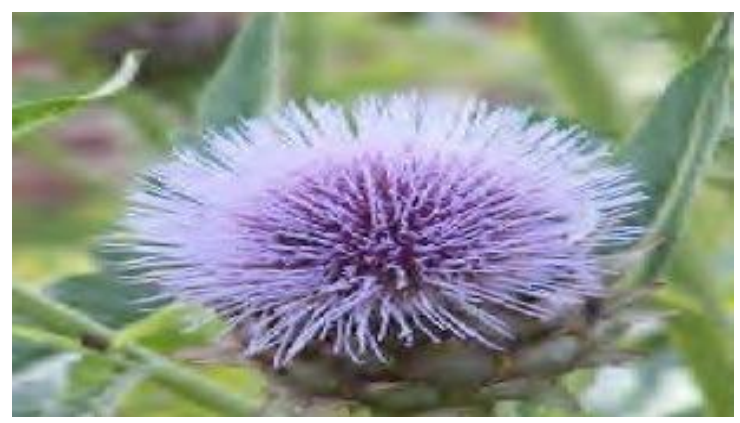

Fig.5. Compressed image by STW method

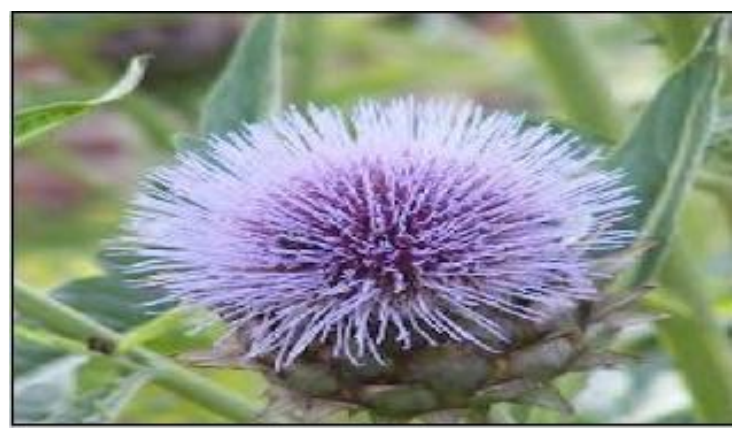

Fig.6. Compressed image by SPIHT method

TABLE NO.1 FOR RESULT COMPARISON

\begin{tabular}{|l|l|l|l|}
\hline & STW & SPIHT & EZW \\
\hline MSE & 0.2046 & 26.85 & 17.36 \\
\hline PSNR & 55.02 & 33.84 & $35.74 \mathrm{~s}$ \\
\hline CR & $3.89 \%$ & $7.85 \%$ & $11.94 \%$ \\
\hline
\end{tabular}

\section{CONCLUSION}

In This paper various schemes for true image compression algorithm based on graphical user interface was put forward, the steps of algorithm and characteristic of 2D wavelet transform are summarized. Simulation results indicate true image compression based on 2D wavelet transform could retain more information of image. 


\section{REFRENCES}

[1] Lewis A S .and Knowles G, 1992 "Image compression using 2-D wavelet transforms "IEEE TRANSACTIONS on image processing.

[2] Sahi, Ramanjit K., 2002 "Image compression using wavelet transforms", Master Theses.

[3] S. Lawson and J. Zhu, 2002 "Image compression using wavelets and jpeg 2000 .

[4] Archana D, G. S. Shirnewar, Dr. A.K. Sahoo, 2005 "A Comparative Study of DCT, DWT \& Hybrid (DCTDWT) Transform," .

[5] M. Angelopoulou, K. Masselos, Peter Cheung, 2006 "A Comparison of 2-D Discrete Wavelet Transform Computation Schedules on FPGAs".

[6] Nageswara Rao Thota and Srinivasa Kumar Devireddy, 2008 "Image Compression Using Discrete Cosine Transform".

[7] Telagarapu P., Jagan N. V. 20011 "Image Compression Using DCT and Wavelet Transformations".
[8] Groach M. Garg A, 2012 "Performance Analysis of DCSPIHT and SPIHT Algorithm for Image Compression".

[9] Jamuna.M, Vijaya Prakash A.M., 2012 "Low Power VLSI Architecture for Image Compression System Using Discrete Wavelet Transform IJEAT".

[10] Mozammel Hoque Chowdhury M. and Khatun Amina, 2012 "Image Compression Using Discrete Wavelet Transform".

[11] Rajesh K. Yadav, S.P. Gangwar, 2012 "Study and analysis of wavelet based image compression techniques".

[12] Bhonde N., Shinde S., 2013 "Image Compression Using Discrete Wavelet Transform",

[13] Sandeep Kaur, Gaganpreet Kaur, 2013 "A Review: Various Wavelet Based Image Compression Techniques".

[14] A.M.Raid, W.M.Khedr, 2014 "Image Compression Using Embedded Zero tree Wavelet".

[15] Vachak Poonam, Nigam Vineeta S. 2014 "Efficient Architecture for Image Compression for Lifting Based Discrete Wavelet Transform,". 